(c) 2010 IEEE. Personal use of this material is permitted. Permission from IEEE must be obtained for all other uses, in any current or future media, including reprinting/republishing this material for advertising or promotional purposes, creating new collective works, for resale or redistribution to servers or lists, or reuse of any copyrighted component of this work in other works. 


\title{
Optimal Cosine Modulated Nonuniform Linear Phase FIR Filter Bank Design via Stretching and Shifting Frequency Response of Prototype Filter
}

\author{
C.Y.F. Ho', Student Member of IEEE, B.W.K. Ling 2 , Senior Member of IEEE, L. Benmesbah ${ }^{2}$, T.C.W. \\ $\mathrm{Kok}^{3}$, Senior Member of IEEE, W.C. Siu ${ }^{4}$, Senior Member of IEEE, and K.L. Teo ${ }^{5}$, Senior Member of IEEE \\ ${ }^{1}$ School of Mathematical Sciences, Queen Mary, University of London, London, United Kingdom \\ 2 Department of Electronic Engineering, King's College London, London, United Kingdom \\ ${ }^{3}$ Canaan Microelectronics, Hong Kong, China \\ ${ }^{4}$ Department of Electronic and Information Engineering, The Hong Kong Polytechnic University, Hong Kong, China \\ ${ }^{5}$ Department of Mathematics and Statistics, Curtin University of Technology, Perth, Australia \\ c.ho@qmul.ac.uk, wing-kuen.ling@kcl.ac.uk, lamia.benmesbah@kcl.ac.uk, eekok@ieee.org, enwcsiu@polyu.edu.hk, \\ K.L.Teo@curtin.edu.au
}

\begin{abstract}
This paper proposes an optimal cosine modulated nonuniform linear phase finite impulse response (FIR) filter bank design. The frequency responses of all the analysis filters and the synthesis filters of the filter bank are derived based on both stretching and shifting the frequency response of the prototype filter. The total aliasing error of the filter bank is minimized subject to a specification on the maximum amplitude distortion of the filter bank as well as specifications on both the maximum passband ripple magnitude and the maximum stopband ripple magnitude of the prototype filter. This filter bank design problem is actually a functional inequality constrained optimization problem. Our recently developed integration approach is employed for solving the problem. Computer numerical simulation results show that our proposed design method outperforms existing design methods.
\end{abstract}

\section{INTRODUCTION}

Filter banks decompose input signals into component signals in different frequency bands. Operations are then applied to these component signals. As the operations could be tailor made according to the frequency characteristics of the component signals, performances of the filter banks are usually very good. Nonuniform filter banks are the filter banks with different decimation factors in different subband channels. This allows the decomposition of input signals into component signals with the component signals having different bandwidths. Hence, nonuniform filter banks enjoy better frequency decomposition compared to uniform filter banks. Finite impulse response (FIR) filter banks are the filter banks with all the analysis filters and the synthesis filters being FIR. As the stability of FIR filters is guaranteed, the stability of the FIR filter banks is also guaranteed. Linear phase filter banks are the filter banks with all the analysis filters and the synthesis filters being linear phase. Since all the analysis filters and the synthesis filters are linear phase, the phase distortion of the filter banks can be avoided. Cosine modulated filter banks are the filter banks that all the analysis filters and the synthesis filters are the cosine modulations of a single prototype filter. As only a single prototype filter is required to be designed, the computational effort for designing the cosine modulated filter banks is usually very low. As a result, cosine modulated nonuniform linear phase FIR filter banks find many applications in various engineering disciplines [1][5].

To design cosine modulated nonuniform FIR filter banks, a single FIR prototype filter is first designed. Then a cosine modulated uniform FIR filter bank is constructed with all the analysis filters and the synthesis filters are the cosine modulations of the FIR prototype filter. Finally, several subband channels of the cosine modulated uniform FIR filter bank are merged together to obtain a cosine modulated nonuniform FIR filter bank [6]-[9]. Similar techniques have been proposed by using more than one single FIR prototype filters [10].

However, even though the FIR prototype filter is linear phase as well as all the analysis filters and the synthesis filters of the cosine modulated uniform FIR filter bank are linear phase [13], it is not guaranteed that all the analysis filters and the synthesis filters of the cosine modulated nonuniform FIR filter bank are linear phase if the cosine modulated nonuniform FIR filter bank is obtained by merging several subband channels of the cosine modulated uniform FIR filter bank together. This is because the filter merged by a symmetric filter and an anti-symmetric filter is not linear phase. Moreover, the amplitude distortion of the cosine modulated nonuniform filter bank as well as both the maximum passband ripple magnitude and the maximum stopband ripple magnitude of the analysis filters and the synthesis filters of the cosine modulated nonuniform filter bank are very large particularly at the transition bands of the analysis filters and the synthesis filters of the cosine modulated uniform filter bank. Hence, the specification on the maximum amplitude distortion of the cosine modulated nonuniform filter bank as well as the specifications on both the maximum passband ripple magnitude and the maximum stopband ripple magnitude of the analysis filters and the synthesis filters of the cosine modulated nonuniform filter bank are usually not satisfied. Furthermore, as the lengths of all the analysis filters and the synthesis filters of the cosine modulated uniform filter bank are the same, the lengths of all the analysis filters and the synthesis filters of 
the cosine modulated nonuniform filter bank are the same too. However, a filter with a wider bandwidth should be designed with a shorter length and vice versa. Hence, these design methods are not effective.

In this paper, a new direct optimal cosine modulated nonuniform linear phase FIR filter bank design is proposed based on both stretching and shifting the frequency response of a single linear phase FIR prototype filter. As stretching the frequency response of the prototype filter is equivalent to sampling the impulse response of the prototype filter if the prototype filter is band limited, the lengths of the analysis filters and the synthesis filters of the filter bank are no longer the same and the lengths are inversely proportional to the bandwidths of the filters. Hence, the proposed stretching technique could effectively design the analysis filters and the synthesis filters of the filter bank. Also, as all the analysis filters and the synthesis filters of the filter bank are derived from both stretching and shifting the frequency response of the prototype filter, both the maximum passband ripple magnitude and the maximum stopband ripple magnitude of all analysis filters and the synthesis filters of the filter bank are only dependent on the prototype filter and the corresponding decimation factors. Hence, by imposing the corresponding constraints on the prototype filter, the specifications on both the maximum passband ripple magnitude and the maximum stopband ripple magnitude of all the analysis filters and the synthesis filters of the filter bank are guaranteed to be satisfied.

Since aliasing of the cosine modulated nonuniform linear phase FIR filter banks could not be canceled if the set of the decimation factors forms an incompatible set [11], the maximum aliasing distortion of the filter banks could be very large. Hence, this paper minimizes the total aliasing error of the filter banks instead of controls the maximum aliasing distortion of the filter banks. In general, the maximum amplitude distortion of filter banks should be kept at a low value. Hence, a constraint is imposed on the maximum amplitude distortion of the filter banks. Also, as filter banks decompose input signals into component signals in different frequency bands, all the analysis filters and the synthesis filters of the filter banks should have good frequency selectivities. As discussed before, both the maximum passband ripple magnitude and the maximum stopband ripple magnitude of the analysis filters and the synthesis filters of the filter banks are dependent only on the prototype filter and the corresponding decimation factors, so constraints are imposed on both the maximum passband ripple magnitude and the maximum stopband ripple magnitude of the prototype filter. These constraints are defined in the frequency domain. However, as the frequency domain is a continuous set and a continuous set consists of an infinite number of elements, the optimization problem consists of an infinite number of constraints. Actually, this cosine modulated nonuniform linear phase FIR filter bank design problem is a functional inequality constrained optimization problem. In general, it is difficult to guarantee that these infinite numbers of constraints would be satisfied. Our recently developed integration approach [12] could be employed for solving the problem.

The rest part of this paper is organized as follows. In Section II, the cosine modulated nonuniform linear phase FIR filter bank design problem is formulated as a functional inequality constrained optimization problem and our recently developed integration approach [12] is employed for solving the problem. In Section III, a design example of the filter banks is illustrated. Finally, a conclusion is drawn in Section IV.

\section{PROBLEM ForMULATION}

Consider an $N$-channel nonuniform filter bank. Denote the impulse responses of the analysis filters and the synthesis filters of the nonuniform filter bank as $h_{i}[n]$ and $f_{i}[n]$ for $i=0,1, \cdots, N-1$, respectively, frequency responses of the analysis filters and the synthesis filters of the nonuniform filter bank as $H_{i}(\omega)$ and $F_{i}(\omega)$ for $i=0,1, \cdots, N-1$, respectively, and the decimators and the upsamplers as $\downarrow n_{i}$ and $\uparrow n_{i}$ for $i=0,1, \cdots, N-1$, respectively. Here, we assume that the sampling factors are positive integers and the nonuniform filter bank is maximally decimated, that is $n_{i} \in \mathrm{Z}^{+}$for $i=0,1, \cdots, N-1$ and $\sum_{i=0}^{N-1} \frac{1}{n_{i}}=1$. The block diagram of the nonuniform filter bank is shown in Figure 1.

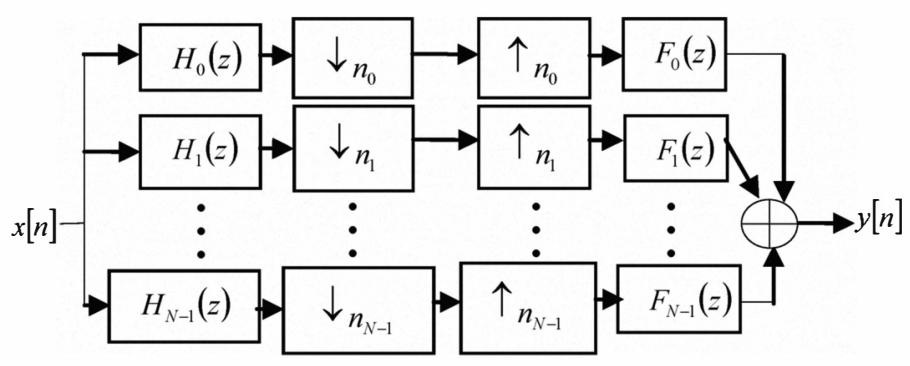

Figure 1. Cosine modulated nonuniform linear phase FIR filter bank.

Denote the impulse response and the frequency response of the linear phase FIR prototype filter as $h^{\prime}[n]$ and $H^{\prime}(\omega)$, respectively. It is assumed that the prototype filter is a lowpass filter with the cutoff frequency $\frac{\pi}{r_{c}}$, where $r_{c}$ is the least common multiple of the set of the scaled sampling factors defined as $r_{c} \equiv \operatorname{LCM}\left(n_{0}, 2 n_{1}, \cdots, 2 n_{N-2}, n_{N-1}\right)$. Since $n_{i} \in \mathrm{Z}^{+}$for $i=0,1, \cdots, N-1, r_{c} \in \mathrm{Z}^{+}$. As the prototype filter is a lowpass linear phase FIR filter, it is assumed that the prototype filter is symmetric. Let the filter length of the linear phase FIR prototype filter be $2 L+1$, where $L$ is an integer multiple of $r_{c}$. Denote the transposition operator as the superscript ${ }^{T}$, the filter coefficients of the prototype filter as $h_{k}^{\prime}$ for $k=0,1, \cdots, 2 L, \mathbf{x} \equiv\left[\begin{array}{lll}h_{0}^{\prime}, \cdots, & h_{L}^{\prime}\end{array}\right]^{T}$ and $\xi(\omega) \equiv[2 \cos (L \omega), \cdots, \quad 2 \cos \omega, 1]^{T}$. Then we have

$$
H^{\prime}(\omega)=e^{-j \omega L}\left(h_{L}^{\prime}+2 \sum_{k=0}^{L-1} h_{k}^{\prime} \cos ((L-k) \omega)\right)=e^{-j \omega L \xi^{T}}(\omega) \mathbf{x}
$$

Since $r_{c}=\operatorname{LCM}\left(n_{0}, 2 n_{1}, \cdots, 2 n_{N-2}, n_{N-1}\right)$, there exist a set of positive integers $q_{i}^{\prime}$ for $i=0,1, \cdots, N-1$ such that $q_{0}^{\prime} n_{0} \equiv r_{c}, q_{N-1}^{\prime} n_{N-1} \equiv r_{c}$ and $2 q_{i}^{\prime} n_{i} \equiv r_{c}$ for $i=1, \cdots, N-2$. Define the sampled, delayed and scaled impulse responses 
of $h^{\prime}[n]$ as $h_{i}^{\prime b}[n] \equiv q_{i}^{\prime} h^{\prime}\left[q_{i}^{\prime} n-L\left(q_{i}^{\prime}-1\right)\right]$, the discrete-time Fourier transform of $h_{i}^{\prime b}[n]$ as $H_{i}^{\prime b}(\omega)$ and

$\xi_{i}^{\prime}(\omega) \equiv\left[2 \cos \left(\frac{L}{q_{i}^{\prime}} \omega\right), \quad 0, \quad \cdots, \quad 0, \cdots, 2 \cos \omega \quad 0, \cdots, \quad 0,1\right]^{T}$ for $i=0,1, \cdots, N-1$. As the time support of $h_{i}^{\prime b}[n]$ is $\left[L\left(1-\frac{1}{q_{i}^{\prime}}\right), L\left(1+\frac{1}{q_{i}^{\prime}}\right)\right]$ for $i=0,1, \cdots, N-1$, we have

$H_{i}^{\prime b}(\omega)=q_{i}^{\prime} e^{-j \omega L}\left(h_{L}^{\prime}+2 \sum_{k=0}^{\frac{L}{q_{i}^{\prime}}-1} h_{q^{\prime}}^{\prime} \cos \left(\left(\frac{L}{q_{i}^{\prime}}-k\right) \omega\right)\right)=q_{i}^{\prime} e^{-j \omega L} \xi_{i}^{\prime T}(\omega) \mathbf{x}^{\cdot}$

As

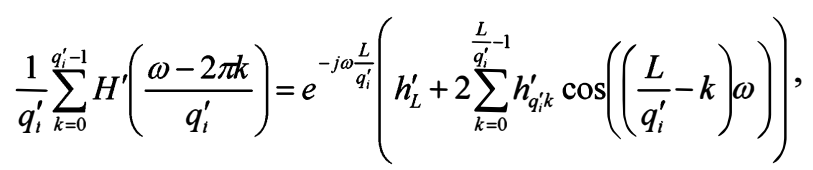

we have

$$
H_{i}^{\prime b}(\omega)=q_{i}^{\prime} e^{-j \omega L} \frac{e^{j \omega \frac{L}{q_{i}^{\prime}}}}{q_{i}^{\prime}} \sum_{k=0}^{q_{i}^{\prime}-1} H^{\prime}\left(\frac{\omega-2 \pi k}{q_{i}^{\prime}}\right) \approx e^{-j \omega L\left(1-\frac{1}{q^{\prime}}\right)} H^{\prime}\left(\frac{\omega}{q_{i}^{\prime}}\right)
$$

for $\omega \in[-\pi, \pi]$ and for $i=0,1, \cdots, N-1$. That is, $H_{i}^{\prime b}(\omega)$ for $i=0,1, \cdots, N-1$ could be approximated by a linear phase modification and stretched version of $H^{\prime}(\omega)$ with the stretched factor $q_{i}^{\prime}$ if $H^{\prime}(\omega)$ is nearly band limited. As the cutoff frequency of $H_{i}^{\prime b}(\omega)$ for $i=0,1, \cdots, N-1$ is located at $\frac{q_{i}^{\prime} \pi}{r_{c}}$ and the bandwidth of $H_{i}^{\prime b}(\omega)$ is $q_{i}^{\prime}$ times that of $H^{\prime}(\omega), H_{i}^{\prime b}(\omega)$ can be interpreted as the corresponding baseband filter of the cosine modulated nonuniform liner phase FIR filter bank.

Let $\phi_{i}$ for $i=0,1, \cdots, N-1$ be some phase angles.

Define $K_{i}=\left\{\begin{array}{ll}1, & i=0 \\ 2, & i=1,2, \cdots, N-2 \\ 1, & i=N-1\end{array} \omega_{i} \equiv\left\{\begin{array}{cc}\frac{\pi}{2 n_{i}}+\sum_{j=0}^{i-1} \frac{\pi}{n_{j}}, & i=1, \cdots, N-2, \\ \pi, & i=N-1\end{array}\right)\right.$ $h_{i}[n] \equiv K_{i} h_{i}^{\prime b}[n] \cos \left(\omega_{i} n+\phi_{i}\right), f_{i}[n]=n_{i} K_{i} h_{i}^{b}[n] \cos \left(\omega_{i} n-\phi_{i}\right)$, $\widetilde{\mathbf{H}}_{i}(\omega) \equiv \frac{K_{i} \cos \phi_{i}}{2}\left(q_{i}^{\prime} e^{-j\left(\omega-\omega_{i}\right) L \xi_{i}^{\prime}}\left(\omega-\omega_{i}\right)+q_{i}^{\prime} e^{-j\left(\omega+\omega_{i}\right) L} \xi_{i}^{\prime}\left(\omega+\omega_{i}\right)\right)$ $+\frac{j K_{i} \sin \phi_{i}}{2}\left(q_{i}^{\prime} e^{-j\left(\omega-\omega_{i}\right) L} \xi_{i}^{\prime}\left(\omega-\omega_{i}\right)-q_{i}^{\prime} e^{-j\left(\omega+\omega_{i}\right) L \xi_{i}^{\prime}}\left(\omega+\omega_{i}\right)\right)$

and

$$
\begin{aligned}
& \widetilde{\mathbf{F}}_{i}(\omega) \equiv \frac{n_{i} K_{i} \cos \phi_{i}}{2}\left(q_{i}^{\prime} e^{-j\left(\omega-\omega_{i}\right) L} \xi_{i}^{\prime}\left(\omega-\omega_{i}\right)+q_{i}^{\prime} e^{-j\left(\omega+\omega_{i}\right) L} \xi_{i}^{\prime}\left(\omega+\omega_{i}\right)\right) \\
& -\frac{j n_{i} K_{i} \sin \phi_{i}}{2}\left(q_{i}^{\prime} e^{-j\left(\omega-\omega_{i}\right) L} \xi_{i}^{\prime}\left(\omega-\omega_{i}\right)-q_{i}^{\prime} e^{-j\left(\omega+\omega_{i}\right) L} \xi_{i}^{\prime}\left(\omega+\omega_{i}\right)\right)
\end{aligned}
$$

for $i=0,1, \cdots, N-1$. Then we have $H_{i}(\omega)=\widetilde{\mathbf{H}}_{i}^{T}(\omega) \mathbf{x}$ and $F_{i}(\omega)=\widetilde{\mathbf{F}}_{i}^{T}(\omega) \mathbf{x}$ for $i=0,1, \cdots, N-1$. Obviously, the centre frequencies of $H_{i}(\omega)$ and $F_{i}(\omega)$ are located at $\omega_{i}$ and $-\omega_{i}$ for $i=0,1, \cdots, N-1$, which are the desirable centre frequencies of the analysis filters and the synthesis filters of the cosine modulated nonuniform linear phase FIR filter bank.

Define

$$
\begin{aligned}
& \mathbf{v}_{i, j}(\omega) \equiv \frac{1}{n_{i}}\left[H_{i}\left(\omega-\frac{2 \pi j}{n_{i}}\right), \quad 0, \cdots, \quad 0\right] \\
& =\frac{1}{n_{i}}\left[\widetilde{\mathbf{H}}_{i}^{T}\left(\omega-\frac{2 \pi j}{n_{i}}\right) \mathbf{x}, \quad 0, \cdots, \quad 0\right]
\end{aligned}
$$

for $i=0,1, \cdots, N-1$ and for $j=0,1, \cdots, n_{i}-1$, where there are $\frac{r_{c}}{n_{i}}-1$ zeros in the vector $\mathbf{v}_{i, j}(\omega)$. Denote

$$
\mathbf{v}_{i}(\omega) \equiv\left[\mathbf{v}_{i, 0}(\omega), \quad \cdots, \quad \mathbf{v}_{i, n_{i}-1}(\omega)\right]^{\prime}
$$

for $i=0,1, \cdots, N-1$ and the aliasing matrix as $\mathbf{V}(\omega)$. Then we have $\mathbf{V}(\omega)=\left[\begin{array}{llll}\mathbf{v}_{0}(\omega), & \mathbf{v}_{1}(\omega), & \cdots, & \mathbf{v}_{N-1}(\omega)\end{array}\right]$. Define

$$
\mathbf{F}(\omega) \equiv\left[\begin{array}{llll}
\tilde{\mathbf{F}}_{0}^{T}(\omega) \mathbf{x}, & \tilde{\mathbf{F}}_{1}^{T}(\omega) \mathbf{x}, & \cdots, & \tilde{\mathbf{F}}_{N-1}^{T}(\omega) \mathbf{x}
\end{array}\right]^{T},
$$

the desirable delay and the desirable overall gain of the cosine modulated nonuniform linear phase FIR filter bank as $m_{0}$ and $c$, respectively, where $m_{0} \in Z^{+}$and $c \in \mathfrak{R}^{+}$. Denote $\boldsymbol{\alpha}(\omega) \equiv\left[\begin{array}{llll}c e^{-j \omega m_{0}}, & 0, & \cdots, & 0\end{array}\right]^{T}$ and $\boldsymbol{\kappa}(\omega) \equiv\left[\begin{array}{llll}\kappa_{0}(\omega), & \kappa_{1}(\omega), & \cdots, & \kappa_{r_{c}-1}(\omega)\end{array}\right]^{T} \equiv \mathbf{V}(\omega) \mathbf{F}(\omega)-\boldsymbol{\alpha}(\omega)$. Then we have

$$
\kappa_{0}(\omega)=\sum_{i=0}^{N-1} \frac{1}{n_{i}} \mathbf{x}^{T} \tilde{\mathbf{H}}_{i}(\omega) \tilde{\mathbf{F}}_{i}^{T}(\omega) \mathbf{x}-c e^{-j o m_{0}}
$$

and

$$
\kappa_{j}(\omega)=\sum_{\substack { i=0 \\
\begin{subarray}{c}{j n_{i} \\
r_{c}{ i = 0 \\
\begin{subarray} { c } { j n _ { i } \\
r _ { c } } }\end{subarray}}^{N-1} \frac{1}{n_{i}} \mathbf{x}^{T} \tilde{\mathbf{H}}_{i}\left(\omega-\frac{2 \pi j}{r_{c}}\right) \tilde{\mathbf{F}}_{i}^{T}(\omega) \mathbf{x}
$$

for $j=1,2, \cdots, r_{c}-1$. Actually, $\left|\kappa_{0}(\omega)\right|$ and $\angle \kappa_{0}(\omega)$ correspond to the amplitude distortion and the phase distortion of the cosine modulated nonuniform linear phase FIR filter bank, respectively, and $\kappa_{j}(\omega)$ for $j=1,2, \cdots, r_{c}-1$ correspond to the aliasing distortion of the cosine modulated nonuniform linear phase FIR filter bank.

To minimize the total aliasing error of the cosine modulated nonuniform linear phase FIR filter bank, $\int_{-\pi}^{\tau} \sum_{j=1}^{r_{c}-1}\left|\kappa_{j}(\omega)\right| d \omega$ is minimized. This is equivalent to $\operatorname{minimize} \int_{-\pi}^{\pi} \sum_{j=1}^{r_{c}-1}\left|\sum_{\substack{i=0 \\ j_{i_{i}} Z^{+} \\ r_{c}}}^{N-1} \frac{1}{n_{i}} \mathbf{x}^{T} \tilde{\mathbf{H}}_{i}\left(\omega-\frac{2 \pi j}{r_{c}}\right) \tilde{\mathbf{F}}_{i}^{T}(\omega) \mathbf{x}\right| d \omega \cdot$ To guarantee the maximum amplitude distortion of the cosine modulated nonuniform linear phase FIR filter bank to be under a certain level, a constraint is imposed on the maximum amplitude distortion of the filter bank. Denote $\delta_{M}$ as the acceptable maximum amplitude distortion of the cosine modulated nonuniform linear phase FIR filter bank. Then we have the constraint $\left|\kappa_{0}(\omega)\right| \leq \delta_{M}$ $\forall \omega \in[-\pi, \pi]$. This is equivalent to $\left|\sum_{i=0}^{N-1} \frac{1}{n_{i}} \mathbf{x}^{T} \widetilde{\mathbf{H}}_{i}(\omega) \widetilde{\boldsymbol{F}}_{i}^{T}(\omega) \mathbf{x}-c e^{-j a m_{0}}\right| \leq \delta_{M} \quad \forall \omega \in[-\pi, \pi] . \quad$ To guarantee all the analysis filters and the synthesis filters of 
the cosine modulated nonuniform linear phase FIR filter bank to have good frequency selectivities, constraints are imposed on the maximum passband ripple magnitude and the maximum stopband ripple magnitude of the prototype filter. Denote $B_{p}$ and $B_{s}$ as the passband and the stopband of the prototype filter, respectively, $\delta_{p}$ and $\delta_{s}$ as the acceptable maximum passband ripple magnitude and the acceptable maximum stopband ripple magnitude of the prototype filter, respectively, and $D(\omega)$ as the desirable magnitude response of the prototype filter. Then we have $\left|\xi^{T}(\omega) \mathbf{x}-D(\omega)\right| \leq \delta_{p} \quad \forall \omega \in B_{p} \quad$ and $\quad\left|\xi^{T}(\omega) \mathbf{x}-D(\omega)\right| \leq \delta_{s}$ $\forall \omega \in B_{s}$. Hence, the cosine modulated nonuniform linear phase FIR filter bank design problem can be formulated as the following functional inequality constrained optimization problem:

Problem (P)

$\min _{\mathbf{x}} \quad \int_{-\pi}^{\pi} \sum_{j=1}^{r_{c}-1}\left|\sum_{\substack{i=0 \\ j n_{i} \in Z^{+} \\ r_{c}}}^{N-1} \frac{1}{n_{i}} \mathbf{x}^{T} \tilde{\mathbf{H}}_{i}\left(\omega-\frac{2 \pi j}{r_{c}}\right) \tilde{\mathbf{F}}_{i}^{T}(\omega) \mathbf{x}\right| d \omega$,

subject to $\left|\sum_{i=0}^{N-1} \frac{1}{n_{i}} \mathbf{x}^{T} \widetilde{\mathbf{H}}_{i}(\omega) \widetilde{\mathbf{F}}_{i}^{T}(\omega) \mathbf{x}-c e^{-j \omega m_{0}}\right| \leq \delta_{M} \quad \forall \omega \in[-\pi, \pi]$,

$$
\left|\xi^{T}(\omega) \mathbf{x}-D(\omega)\right| \leq \delta_{p} \quad \forall \omega \in B_{p}
$$

and

$$
\left|\xi^{T}(\omega) \mathbf{x}-D(\omega)\right| \leq \delta_{s} \quad \forall \omega \in B_{s} .
$$

This functional inequality constrained optimization problem could be solved via our recently proposed integration approach [12].

\section{ILLUSTRATIVE EXAMPLE}

As discussed in Section III that the maximum aliasing distortion of the cosine modulated nonuniform filter bank is very large if the set of the decimation factors forms an incompatible set [11], the cosine modulated nonuniform filter bank with an incompatible set of the decimation integers $\{2,3,6\}$ is designed to illustrate the effectiveness of the proposed method. In general, there is a tradeoff among the length, the transition band bandwidth, the maximum passband ripple magnitude and the maximum stopband ripple magnitude of the prototype filter as well as the maximum amplitude distortion of the cosine modulated nonuniform linear phase FIR filter bank. Hence, we choose $L=120$

$B_{p}=\left[0.025 \pi-\frac{\pi}{6}, \frac{\pi}{6}-0.025 \pi\right]$

$B_{s}=\left[\frac{\pi}{6}+0.025 \pi, \pi\right] \bigcup\left[-\pi,-\frac{\pi}{6}-0.025 \pi\right], \delta_{p}=0.0869$,

$\delta_{s}=0.2068, D(\omega)=\left\{\begin{array}{ll}1 & \omega \in B_{p} \\ 0 & \omega \in B_{s}\end{array}\right.$ and $\delta_{M}=0.0505$, which

are the most common values employed in the practical applications [1]-[5]. In order to achieve cosine modulated nonuniform linear phase FIR filter banks, $\phi_{i}=0$ for $i=0,1, \cdots, N-1$ are selected [13]. The desirable overall gain of the cosine modulated nonuniform linear phase FIR filter bank is selected as $c=1$ for a simplicity reason. Since the minimum delay of the cosine modulated nonuniform linear phase FIR filter bank is equal to $2 L$, the desirable delay of the cosine modulated nonuniform linear phase FIR filter bank is selected as $m_{0}=2 L$. Based on the formulation discussed in Section II and the integration approach discussed in [12], the cosine modulated nonuniform linear phase FIR filter bank could be designed.

A cosine modulated nonuniform FIR filter bank obtained by merging the subband channels [9] of the corresponding cosine modulated uniform linear phase FIR filter bank together [13] is designed for the comparison purpose. In order to have a fair comparison, the design based on the methods discussed in [13] and [9] is also formulated as a functional inequality constrained optimization problem with the same set of cost function and constraint functions as those discussed in Section II. Figures 2, Figure 3, Figure 4, Figure 5 and Figure 6 show the responses of the prototype filters, the responses of the analysis filters, the responses of the synthesis filters, the amplitude distortions of the filter banks, and the aliasing distortions of the filter banks, respectively. It can be seen from those figures that the specifications on both the maximum passband ripple magnitude and the maximum stopband ripple magnitude of the prototype filter as well as the specification on the maximum amplitude distortion of the filter bank designed using our proposed method are satisfied. In fact, the maximum passband ripple magnitude and the maximum stopband ripple magnitude of the prototype filter designed based on our proposed method could achieve 0.0869 and 0.2068 , respectively, and the maximum amplitude distortion of the filter bank designed based on our proposed method could achieve 0.0505 . On the other hand, the specifications on the maximum passband ripple magnitude and the maximum stopband ripple magnitude of the prototype filter as well as the specification on the maximum amplitude distortion of the filter bank designed based on the methods discussed in [13] and [9] are not satisfied. In fact, the maximum passband ripple magnitude and the maximum stopband ripple magnitude of the prototype filter designed based on the methods discussed in [13] and [9] could only achieve 0.1063 and 0.2619 , respectively, and the maximum amplitude distortion of the filter bank designed based on the methods discussed in [13] and [9] could only achieve 0.1446 . Moreover, both the maximum passband ripple magnitude and the maximum stopband ripple magnitude of the analysis filters and the synthesis filters as well as the maximum aliasing distortion of the filter bank designed based on our proposed method are much lower than that designed based on the methods discussed in [13] and [9]. Furthermore, it is worth noting that the analysis filters and the synthesis filters of the filter bank designed based on the methods discussed in [13] and [9] are not linear phase, while our design guarantees that all the analysis filters and the synthesis filters are linear phase. 


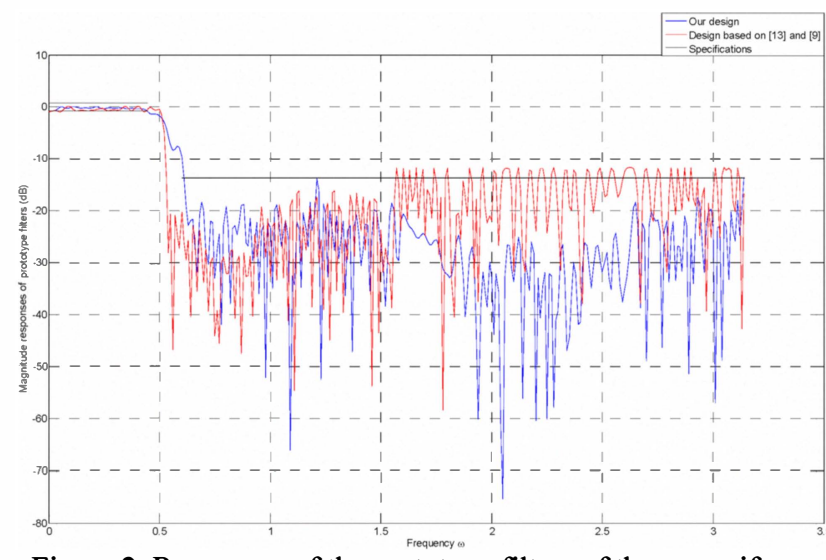

Figure 2. Responses of the prototype filters of the nonuniform filter banks.

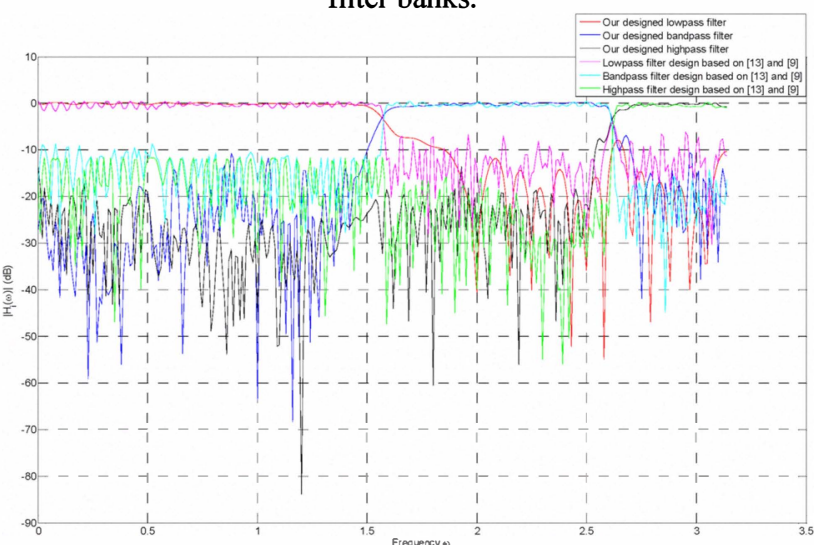

Figure 3. Responses of the analysis filters of the nonuniform filter banks.

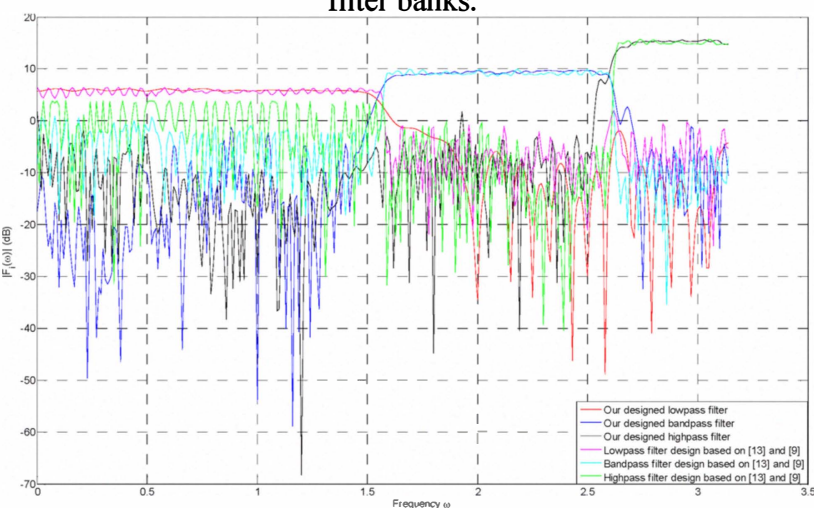

Figure 4. Responses of the synthesis filters of the nonuniform filter banks.

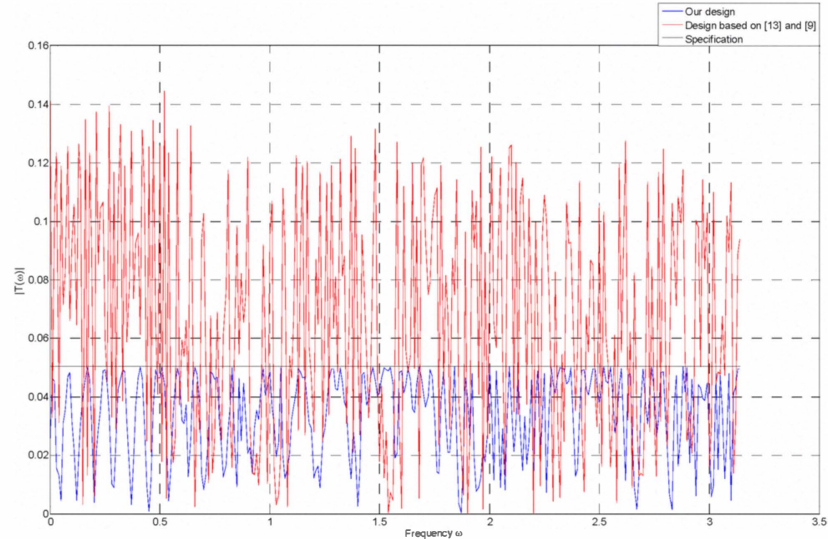

Figure 5. Amplitude distortions of the nonuniform filter banks.
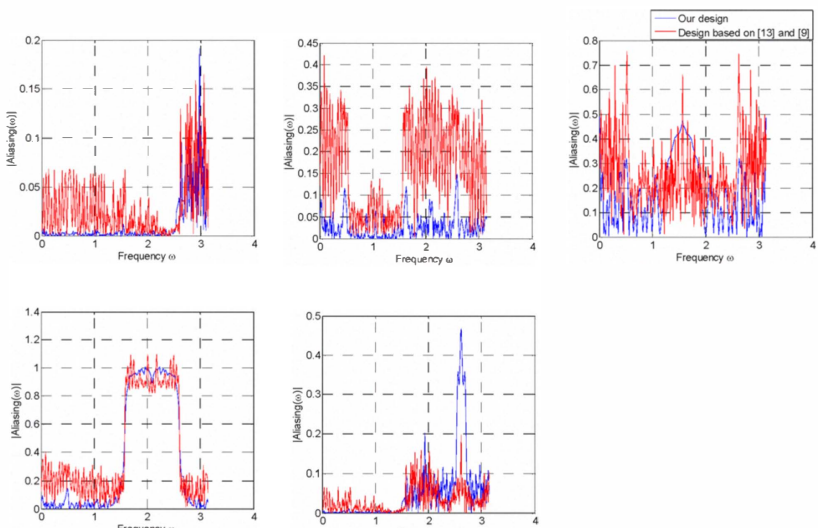

Figure 6. Aliasing distortions of the nonuniform filter banks.

\section{CONCLUSION}

This paper proposes a new direct optimal cosine modulated nonuniform linear phase FIR filter bank design based on both stretching and shifting the frequency response of a single linear phase FIR prototype filter. The total aliasing error is minimized subject to a specification on the maximum amplitude distortion of the filter bank as well as both the maximum passband ripple magnitude and the maximum stopband ripple magnitude of the prototype filter. The design problem is actually a functional inequality constrained optimization problem and our recently proposed integration approach is employed for solving the problem. As the length of all the analysis filters and the synthesis filters are inversely proportional to the bandwidth of the filters, the proposed design method is more effective. Also, as the proposed design method is a direct design method, merging the subband channels of the cosine modulated uniform filter bank together is not required. Hence, our design could achieve a better performance on the maximum amplitude distortion of the filter bank as well as better performances on both the maximum passband ripple magnitude and the maximum stopband ripple magnitude of the prototype filter.

\section{ACKNOWLEDGMENTS}

The work obtained in this paper was supported by a research grant (project number G-YD26) from The Hong Kong Polytechnic University, the Centre for Multimedia Signal Processing, The Hong Kong Polytechnic University, the CRGC grant (project number PolyU $5105 \backslash 01 \mathrm{E})$ from the Research Grants Council of Hong Kong and a research grant from the Australian Research Council.

\section{REFERENCES}

[1] Fabrizio Argenti and Enrico Del Re, "Eigenfilter design of real and complex coefficient QMF prototypes," IEEE Transactions on Circuits and Systems -II: Analog and Digital Signal Processing, vol. 47, no. 8, pp. 787-792, 2000.

[2] Jacob D. Griesbach, Michael Lightner and Delores M. Etter, "Subband adaptive filtering decimation constraints for oversampled nonuniform filterbanks," IEEE Transactions on Circuits and Systems -II: Analog and Digital Signal Processing, vol. 49, no. 10, pp. 677-681, 2002.

[3] Ng Chun Kiam and B. Farhang-Boroujeny, "New results on subband adaptive filters with nonuniform filter banks," IEEE Region 10 Conference, TENCON, vol. 2, pp. 1010-1013, 15-17 September 1999. 
[4] Yin H. Lam and Robert Stewart, "Perceptron-based residual analysis-synthesis system," IEEE Conference on Acoustics, Speech, and Signal Processing, ICASSP, vol. 2, pp. 989-992, 1519 March 1999.

[5] Unto K. Laine and Toomas Altosaar, "An orthogonal set of frequency and amplitude modulated (FAM) functions for variable resolution signal analysis," IEEE Conference on Acoustics, Speech, and Signal Processing, ICASSP, vol. 3, pp. 1615-1618, 36 April 1990.

[6] X. M. Xie, S. C. Chan and T. I. Yuk, "Design of perfectreconstruction nonuniform recombination filter banks with flexible rational sampling factors," IEEE Transactions on Circuits and Systems - I: Regular Papers, vol. 52, no. 9, pp. 1965-1981, 2005.

[7] S. S. Yin, S. C. Chan, K. M. Tsui and X. M. Xie, "On the theory and design of a class of PR uniform and recombination nonuniform casual-stable IIR cosine modulated filter banks," IEEE Transactions on Circuits and Systems -II: Express Briefs, vol. 55, no. 8, pp. 776-780, 2008.

[8] Jeong-Jin Lee and Byeong Gi Lee, "A design of nonuniform cosine modulated filter banks," IEEE Transactions on Circuits and Systems -II: Analog and Digital Signal Processing, vol. 42, no. 11, pp. 732-737, 1995.

[9] Jianlin Li, Troung Q. Nguyen and Sawasd Tantaratana, "A simple design method for near-perfect-reconstruction nonuniform filter banks," IEEE Transactions on Signal Processing, vol. 45, no. 8, pp. 2105-2109, 1997.

[10] Fabrizio Argenti, Benedetto Brogelli and Enrico Del Re, "Design of pseudo-QMF banks with rational sampling factors using several prototype filters," IEEE Transactions on Signal Processing, vol. 46, no. 6, pp. 1709-1715, 1998.

[11] Phuong-Quan Hoang and P. P. Vaidyanathan, "Non-uniform multirate filter banks: theory and design," IEEE International Symposium on Circuits and Systems, vol. 1, pp. 371-374, 8-11 May 1989.

[12] Charlotte Yuk-Fan Ho, Bingo Wing-Kuen Ling, Yan-Qun Liu, Peter Kwong-Shun Tam and Kok-Lay Teo, "Optimal design of magnitude responses of rational infinite impulse response filters," IEEE Transactions on Signal Processing, vol. 54, no. 10, pp. 4039-4046, 2006.

[13] Yuan-Pei Lin and P. P. Vaidyanathan, "Linear phase cosine modulated maximally decimated filter banks with perfect reconstruction," IEEE Transactions on Signal Processing, vol. 42, no. 11, pp. 2525-2539, 1995. 